\title{
Detection of Hydrogen Fluoride Gas Using Piezoresistive Microcantilever Sensors
}

\author{
T. L. Porter*, T. Vail ${ }^{1}$, R. Stewart ${ }^{2}$ and J. Reed ${ }^{2}$ \\ Northern Arizona University, Dept of Physics, Box 6010, Flagstaff, AZ 86011, USA \\ ${ }^{1}$ Northern Arizona University, Dept of Chemistry, Box 5698, Flagstaff, AZ 86011, USA \\ ${ }^{2}$ Cantimer, Inc., 3700 Haven Court, Menlo Park, CA, 94025, USA
}

(Received October 4, 2007; accepted January 11, 2008)

Key words: keratin, hydrogen fluoride, microcantilever

We have used embedded piezoresistive microcantilever (EPM) sensors for the detection of hydrogen fluoride (HF) gas. These sensors have a keratin-based compound as the primary sensing material. Exposures to HF at high levels (beginning at $0 \mathrm{ppm}$ and leveling off at $2300 \mathrm{ppm}$ ) resulted in nearly immediate response. Low-level exposures beginning at $0 \mathrm{ppm}$ and leveling off at $230 \mathrm{ppm}$ required $15 \mathrm{~s}$ for a measurable sensor response.

\section{Introduction}

Hydrofluoric acid (HF) is a strong acid used for many purposes in a variety of industries such as the petroleum industry, semiconductor processing, pharmaceutical, glass, hospital, and nuclear industries. For example, of the 148 refineries operating in the United States, 50 use and store large amounts of HF, over 10 million pounds in total. Exposure to HF causes extreme burns of the skin, however, high levels of pain may not be felt for up to $24 \mathrm{~h}$. If not treated, exposed areas may continue to be damaged for many days, resulting in deep tissue damage. Once inside the body, HF can react with the magnesium and calcium in the blood, removing calcium and resulting in a condition known as hypocalcaemia. Hydrogen fluoride gas (HF vapor) may be inhaled as well, causing damage to the lungs and throat. Inhaled in large amounts, HF can cause cyanosis, and eventually, pulmonary edema.

There are a number of current detection technologies capable of indicating the presence of HF gas. Electrochemical detectors can signal the presence of HF (90\%) in $30 \mathrm{~s}$ at levels of $10 \mathrm{ppm}^{(1)}$ Many of these electrochemical detectors are also crosssensitive to other vapors, such as $\mathrm{HCl}(\mathrm{HCL}), \mathrm{Cl}_{2}, \mathrm{NO}_{2}$ and $\mathrm{SO}_{2}$. $\mathrm{HF}$ may also be

*Corresponding author: e-mail: Tim.Porter@nau.edu 
detected by colorimetric means, at levels around $20 \mathrm{ppm} .{ }^{(2)}$ These detectors are small and portable, but may take more time to respond to low levels of HF. Optical methods such as infrared may also be used; however, these systems are generally large and expensive. Other techniques such as diode-laser detection, ${ }^{(3,4)}$ tin-dioxide based gas sensors, ${ }^{(5)}$ surface acoustic wave (SAW) devices, ${ }^{(6)}$ and Si interferometry(7) are also available and currently in use (diode-laser). Mertens et al. have used a microcantilever functionalized with $\mathrm{Si}_{3} \mathrm{~N}_{4}$ or $\mathrm{SiO}_{x}$ layers to detect trace amounts of $\mathrm{HF}$ in air using vibrational modes in the cantilever. ${ }^{(8)}$

In this paper, we discuss the detection of HF gas using embedded piezoresistive microcantilever (EPM) sensors. ${ }^{(9)}$ EPM sensors provide a simple, low-cost and effective platform for the detection of several types of analytes. In the basic EPM sensor, a piezoresistive microcantilever is embedded or partially embedded into a sensing material. The sensing material is typically a polymer, composite polymer, biological material or other composite that acts as a probe for the desired analyte. Upon analyte exposure, chemical, physical or other reactions with the sensor material result in a volumetric change in the sensing material, which is transduced by strain in the cantilever and ultimately measured as a resistance change. The volumetric shift in the sensing material may be due to several mechanisms. These mechanisms might include diffusion of the analyte molecules into the sensing material, probe-target binding on the material surface or bulk, or surface or bulk chemical reactions between the analyte and sensing material. Cantilever strains of only a few angstroms are potentially measurable. Electronics for EPM sensors are simple, as only the cantilever resistance is measured during a sensing event. A simple Wheatstone bridge circuit is sufficient for most measurements. Previously, we tested EPM sensors in a variety of sensing applications with a various analytes. These applications include the sensing of volatile organic compounds (VOC's), ${ }^{(10-13)}$ chlorinated hydrocarbons in water and vapor phase, personal hydration monitoring, ${ }^{(14)}$ carbon monoxide gas, ${ }^{(15)}$ single-strand DNA detection, ${ }^{(16)}$ protein detection, ${ }^{(17)}$ and viral detection. ${ }^{(18)}$

\section{Materials and Methods}

The microcantilevers used in these experiments were designed by Cantimer, Inc., Menlo Park, CA. The piezoresistive microcantilevers are approximately 200 microns in length and 40 microns wide. The cantilevers extend into a small circular area on each die to contain the sensing material and also to protect the cantilever during handling. Each cantilever die also contains an integrated thermistor to be used for temperature correction in applications where it is needed. Figure 1(a) shows a photograph of a single cantilever die. For sensor assembly, a measured amount of sensing material is laid onto the edge of a Si substrate. The liquid material size and volume are controlled by a precision dispensing unit. While the polymer is still wet, the microcantilever tip is positioned to contact the polymer with a micromanipulator. The substrate is then bonded to the chip using epoxy. A graphical representation of the sensor assembly process is shown in Fig. 1(b). The nominal resistance of the bare cantilevers before assembly was $2.2 \mathrm{k} \Omega$. After material deposition and drying, the finished sensors exhibited resistances 
(a)

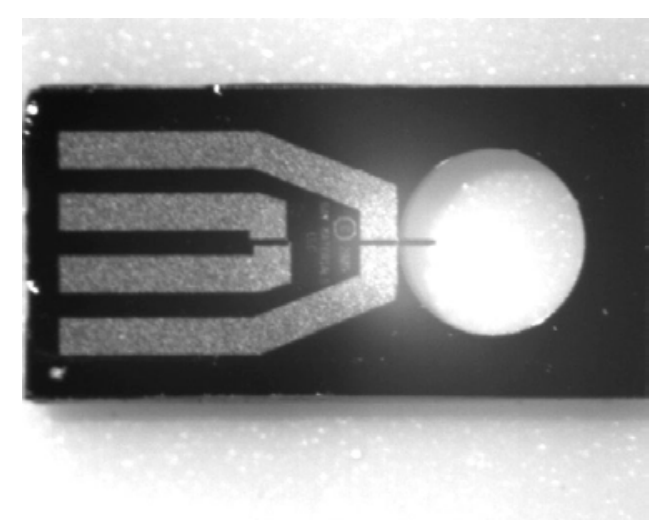

(b)

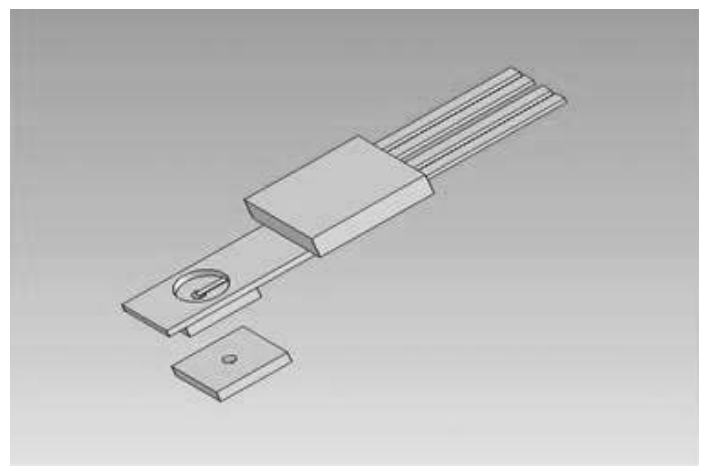

Fig. 1. (a) Photograph of a single cantilever die assembly. The piezoresistive microcantilever extends partially into the circular area on the die. There are four $\mathrm{Al}$ traces on the die, two for the microcantilever, and two for the integrated thermistor. (b) Graphical representation of the sensor assembly process. Here, the microcantilever has been partially embedded into the sensing material deposited on the substrate seen below the die. Also, four larger wire leads have been attached to the full sensor assembly.

of approximately $2.15 \mathrm{k} \Omega$, indicating that the cantilevers have been prestrained by an amount equivalent to 50 Ohms.

The sensing material used in these experiments consisted of a keratin matrix with thiolated colloidal gold particles mixed in. Keratins are long protein chains containing a high percentage (15-17\%) of the amino acid cysteine, required for the disulfide bridges that give strength and rigidity by permanent, stable crosslinking. Keratins also contain a high proportion of the smallest of the 20 amino acids, glycine, whose side group is a single hydrogen atom; also the next smallest, alanine, with a noncharged methyl group. The supramolecular assembly of $\alpha$-keratins includes multiple disulfide cross-links that form intermediate filaments. ${ }^{(19)}$ We mixed keratin with beta-mercaptoethanol to reduce disulfides to sulfhydryls, and then with colloidal gold to form a self-assembled matrix of nanogold-keratin via gold thiolate coordination. The keratin used was VariKer 100 
keratin powder (Variati and Company, Milan Italy), while the colloidal gold was 20-nmdiameter particles in aqueous suspension, used as supplied (Sigma-Aldrich, St. Louis, MO). The ratio of the two components in this material was 1:1. Other sensors were fabricated using the keratin only, with no addition of thiolated gold. The results for both types of sensing materials were virtually identical, indicating that the primary sensing reaction was with the keratin alone.

In this study, we use a single-chip AD7793 24-bit A-D converter which functions as a $6 \frac{1}{2} 2$ digit multimeter to directly measure the cantilever resistance using two leads. The AD7792 chip is interfaced to a laptop computer through a USB interface provided by a USBmicro 421 chip. The data collection is performed on the laptop computer using LabView (National Instruments, Austin, TX). Figure 2(a) shows a photograph of an assembled cantilever die (at the end of the three-inch-long flexible circuit board) and Fig.

(a)

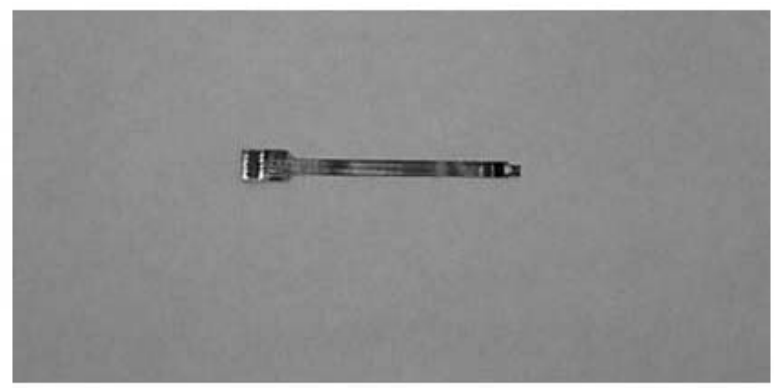

(b)

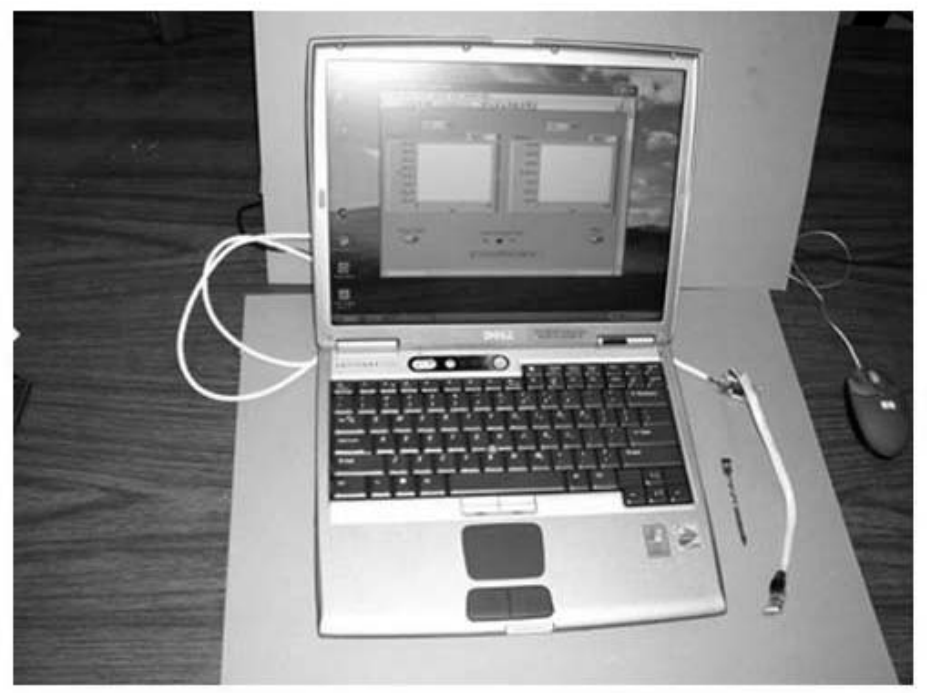

Fig. 2. (a) Photograph of an assembled cantilever die at the end of the three-inch long flexible circuit board. The microcantilever die (sensor) is attached to the right end of the flexible board. (b) Photograph of the entire sensing apparatus, including interface circuitry and laptop computer. The AD7793 amplifier and A-D converter are seen at the lower end of the white cable to the right of the laptop computer. 
2(b) a photograph of the entire sensing apparatus, including interface circuitry and laptop computer. Testing of the sensors took place in a chamber of approximately $3.5 \mathrm{l}$ volume. For the sensor exposures, a measured amount of concentrated liquid hydrofluoric acid was injected into the chamber and allowed to vaporize naturally. During exposure, there was no flow of air in or out of the chamber in order to better quantify the HF concentration in the chamber. After exposure, the chamber was vented by dry nitrogen flow.

\section{Results and Discussion}

Figure 3 shows the results of the first HF exposure. In this first exposure, $1 \mathrm{ml}$ of concentrated HF solution was injected into the test chamber. For this exposure, we estimate the actual HF vapor concentration in the chamber to rise from zero to a

(a)

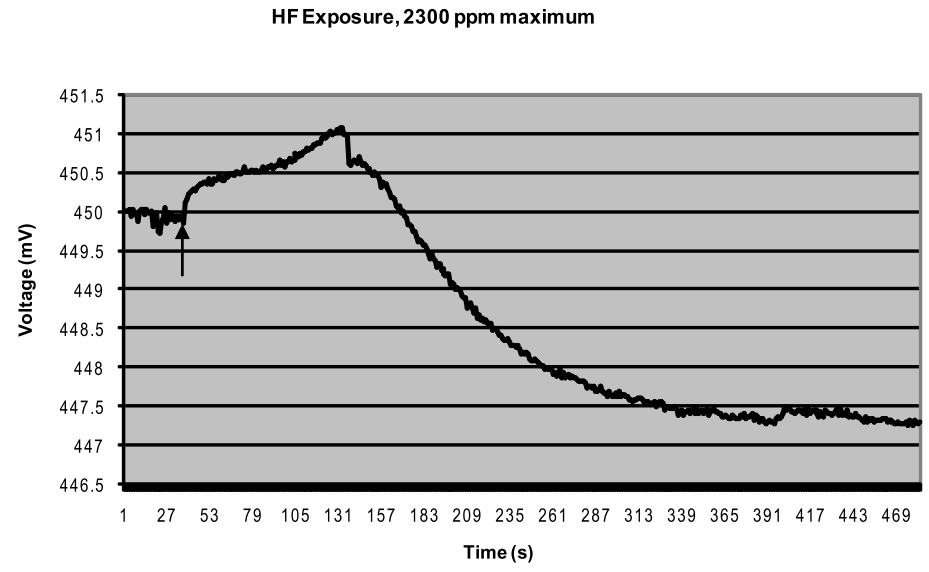

HF Exposure, 2300 ppm maximum

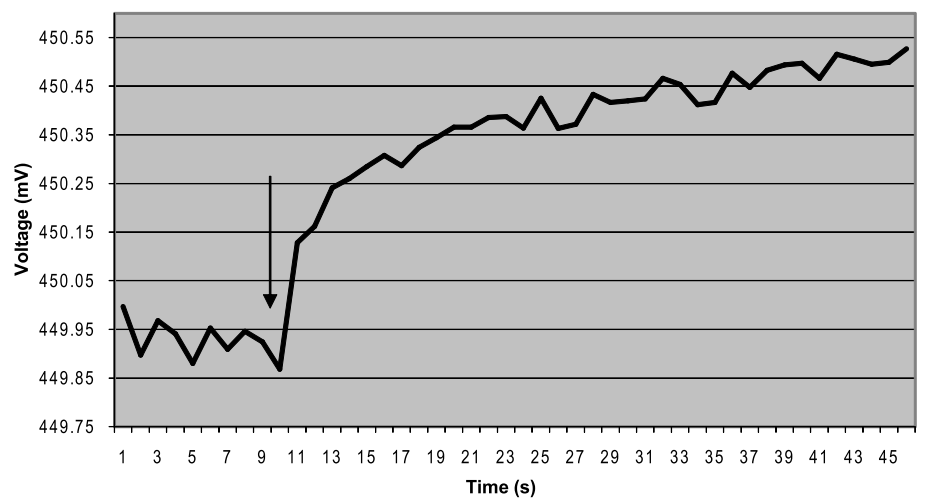

Fig. 3. $2300 \mathrm{ppm}$ maximum exposure to HF. Full exposure of $8 \mathrm{~min}$ (a) and $46 \mathrm{~s}$ interval from $10 \mathrm{~s}$ prior to exposure to $36 \mathrm{~s}$ after the exposure (b). 
maximum of $2300 \mathrm{ppm}$ during the 8 min exposure period. This estimate is based upon the estimated volume of the initial amount injected that evaporated during the time interval. In Fig. 3(a), the full exposure of 8 min is shown, while in Fig. 3(b) only the 46 $\mathrm{s}$ interval from $10 \mathrm{~s}$ prior to exposure to $36 \mathrm{~s}$ after the exposure is shown. The exact time of initial exposure is indicated by the arrows in both graphs. In both graphs, we see that the initial EPM sensor response to the HF is almost immediate, occurring within a few seconds of exposure. From the top graph in Fig. 3, we can see that initially the sensor voltage increases, indicating that the cantilever was "unloading" some of the initial prestrain during the sensor assembly process. This corresponds to the cantilever bending back toward the bottom substrate somewhat. After the initial rise in sensor voltage, the voltage (and thus the cantilever) abruptly reverses direction, indicating that the cantilever was bending toward the original prestrain direction, upward or away from the bottom substrate. The net voltage change from the highest reading to the end was approximately 3.8 $\mathrm{mV}$. With a cantilever sensitivity of approximately $4.7 \Omega / \mu \mathrm{m}$, we estimate, on the basis of the current passing through the cantilever, that the net cantilever deflection from prior to exposure to end of exposure was $3.7 \mu \mathrm{m}$. The full EPM sensor response to the HF analyte, in which the initial response was a rapid downward movement of the cantilever, followed by a larger upward deflection was observed by us previously in multiple systems that respond rapidly to the analyte. ${ }^{(13,19)}$ Current models describing the diffusion or partitioning of analyte molecules into polymer or other host matrices do not predict this behavior. ${ }^{(20)}$ One possibility is that two separate expansion/contraction mechanisms occur within the sensing layer. Upon initial HF exposure, a rapid, volume-decreasing mechanism, which is largely confined to the sensing material surface, occurs first. This initial response may be attributable to the rapid protonation of the keratin by the HF, breaking keratin disulfide cross-links. The second, slower and ultimately larger response may be due to bulk diffusion and subsequent chemical reaction of the HF molecules with the host sensing material. After 8 min of exposure, the chamber was opened, and the HF liquid neutralized. The HF was still very active during neutralization, indicating that the HF gas was continuing to volatilize throughout the experiment. Thus, the sensor exposure to HF was not constant, but rising throughout the experiment. After the experiment, the same sensor was re-exposed to a similar amount of HF in an identical experiment. Virtually no response was recorded, indicating that the HF vapor (at this high concentration) had rendered the sensing layer inoperative.

These keratin-based sensors have also been previously exposed to water vapor alone. The sensor response from an exposure to water vapor similar to that which occurred in the HF exposure produces only a tiny response, less than $1 / 100$ th that of the HF solution. Also, the water vapor exposures do not result in the abrupt cantilever direction changes; instead they show a slow gradual expansion that is fully reversible upon removal of the water vapor.

In the next set of experiments, the amount of concentrated HF liquid injected into the chamber was reduced to $0.1 \mathrm{ml}$, or one-tenth of the previous amount. Here, the HF vapor exposure is assumed to rise from zero (at the time indicated by the arrow) to a maximum value of $230 \mathrm{ppm}$ by the end of the test. As with the previous tests, the HF solution was still very active at the end of the test, indicating continuous vaporization 
of HF. Figure 4 shows the sensor response to this smaller amount of HF. From Fig. 4, we see that the initial rise in sensor voltage is slower, taking over $15 \mathrm{~s}$ to show a clear upward trend. Also, at this lower level of exposure, the sensor voltage continued to move upward slowly throughout the experiment time of $8 \mathrm{~min}$, with no voltage reversal as seen at the higher exposure level (Fig. 3(a)). The total rise in sensor voltage for the $8 \mathrm{~min}$ exposure interval is approximately $0.22 \mathrm{mV}$, considerably less than the $1.0 \mathrm{mV}$ rise observed during the first, higher exposure prior to the sensor response reversing direction and rapidly dropping. We also performed two additional tests using the same sensor previously exposed to the $230 \mathrm{ppm}$ level of HF. These two tests indicated that the sensor could continue to respond to additional exposures (230 ppm or less) of HF, with approximately similar voltage changes. Because the HF damage to the keratin sensing layers is cumulative, we expect that eventually all sensors will be "exhausted" by continued exposure to HF.

(a)

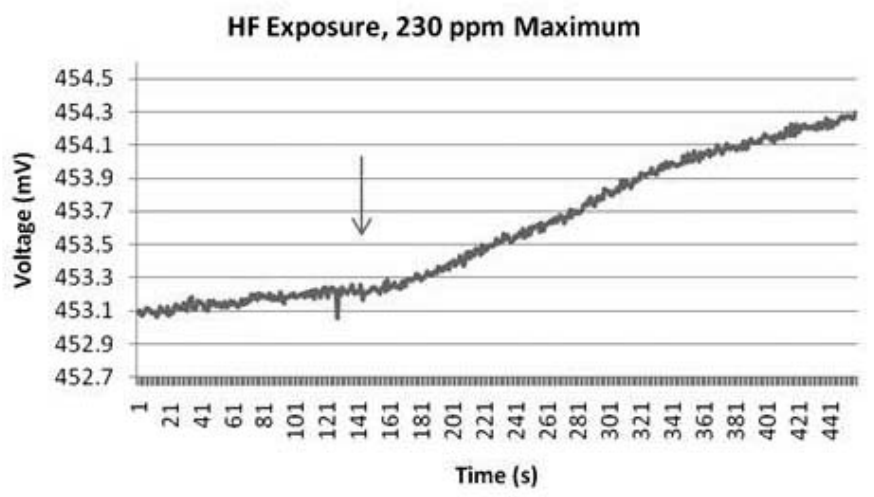

(b)

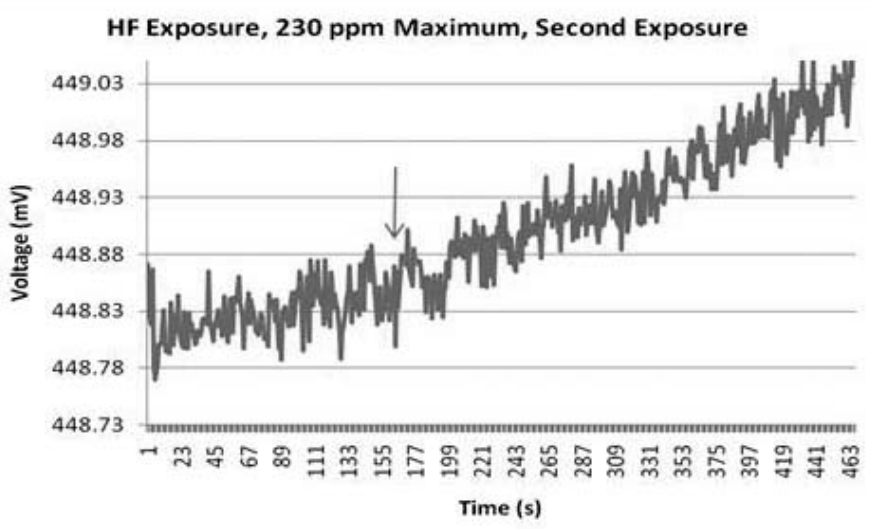

Fig. 4. Sensor response to $230 \mathrm{ppm}$ maximum exposure to HF. 
Finally, we tested the HF sensors to the vapors from sulfuric acid. Here, the sensor response was negligible indicating that the chemical reaction occurring during exposure to $\mathrm{HF}$ is not occurring during exposure to sulfuric acid vapor exposure.

\section{Conclusions}

Embedded piezoresistive microcantilever sensors may be easily fabricated using keratin compounds as the primary sensing material. This sensing material will respond to HF gas quickly (within $15 \mathrm{~s}$ ) at concentrations in the range of 230-2300 ppm. At high concentrations, the sensing material is permanently damaged by the HF, while low HF exposures allow the sensing units to respond to subsequent HF exposures.

\section{References}

1 G. D. MSA Inc. (Pittsburgh, USA).

2 N. F. S. RAE Systems (San Jose, USA).

3 K. Cerf and G. Krieg: Monitoring of Gaseous Pollutants by Tunable Diode Lasers (Reidel, New York, 1987).

4 K. L. McNesby, R. R. Skags, A. W. Miziolek, M. Clay, S. H. Hoke and C. S. Miser: Applied Physics B 67 (1998) 443.

5 J. B. Sanchez, F. Berger, M. Fromm, M. H. Nadal and V. Eyraud: Thin Solid Films 436 (2003) 132.

6 V. B. Patissier, G. Lengaigne, W. Daniau, G. Martin, P. Blind, D. Hauden, M.-H. Nadal and S. Ballandras: IEEE Transactions on Ultrasonics, Ferroelectronics and Frequency Control 54 (2007) 1687.

7 S. E. Letant and M. J. Sailor: Advanced Materials 12 (2000) 355.

8 J. Mertens, E. Finot, M.-H. Nadal, V. Eyraud, O. Heintz and E. Bourillot: Sens. Actuators B 99 (2004) 58.

9 T. L. Porter, M. P. Eastman, C. Macomber, W. G. Delinger and R. Zhine: Ultramicroscopy 97 (2003) 365.

10 T. L. Porter, W. Delinger and R. L. Gunter: Proc. Materials Research Soc. (2005).

11 T. L. Porter, M. P. Eastman, D. L. Pace and M. Bradley: Sens. Actuators A 88 (2001) 47.

12 T. L. Porter, M. P. Eastman, D. L. Pace and M. Bradley: Scanning 22 (2000) 1.

13 R. L. Gunter, W. Delinger, T. L. Porter, R. Stewart and J. Reed: Medical Engineering and Physics 27 (2005) 215.

14 A. Kooser, R. L. Gunter, W. G. Delinger, T. L. Porter and M. P. Eastman: Sens. Actuators 99 (2004) 430.

15 R. L. Gunter, R. Zhine, W. Delinger, K. Manygoats, A. Kooser and T. L. Porter: IEEE Sensors 4 (2004) 430.

16 A. Kooser, K. Manygoats, M. P. Eastman and T. L. Porter: Biosensors and Bioelectronics 19 (2003) 503.

17 R. L. Gunter, W. G. Delinger, K. Manygoats, A. Kooser and T. L. Porter: Sens. Actuators A 107 (2003) 219.

18 D. L. Nelson and M. M. Cox: Principles of Biochemistry (W. H. Freeman and Co., New York, 2005).

19 T. L. Porter, T. Vail, M. P. Eastman, R. Stewart, J. Reed, R. Venedam and W. Delinger:Sens. Actuators 123 (2007) 313.

20 E. L. Cussler: Diffusion-Mass Transfer in Fluid Systems, 2nd ed. (Cambridge University Press, Cambridge, United Kingdom, 1997). 